\title{
Automatic Brain MR Image Lesion Segmentation using Artificial Bee Colony Optimization Algorithm
}

\author{
D. Janaki Sathya \\ Assistant Professor \\ EEE Department \\ PSG College of Technology
}

\begin{abstract}
Medical image segmentation is a very important part of computer assisted diagnostic tools. The brain MR images segmentation is a complex and challenging task. However, precise segmentation of these MR images is very significant for detecting lesions. Segmentation of MR images may assist in tumor diagnosis and treatment by tracking the progress of tumor growth. The Magnetic Resonance Imaging has been proved to provide high resolution medical images and is widely used especially for brain. In this paper, a novel clustering using the swarm intelligence algorithm is presented for the segmentation of brain MR images, intensity - based segmentation using artificial bee colony clustering has been implemented. Statistical tests performed on both real and simulated brain MR images shows good results, which ensures the application of this segmentation algorithm to different medical images and further investigation.
\end{abstract}

\section{Keywords}

Artificial swarm intelligence, Brain MR images, Lesion segmentation, Edge enhancement.

\section{INTRODUCTION}

The Image segmentation holds a position of great importance in the field of image processing. The role of segmentation in case of medical image segmentation is to analyse the anatomical structure, detect region of interest i.e. locate lesion, measure tissue volume to measure growth of the lesion, helps in treatment planning earlier to radiation therapy and in radiation dose calculation and other abnormalities. Brain MR Image segmentation is a significant and rapidly developing area of research for long time. There are many medical segmentation algorithms [1-5] but there is no such generic algorithm for providing absolutely successful segmentation of medical images. It becomes more significant while typically dealing with medical images were pre-surgery and post-surgery analysis are required for the purpose of instigating and speeding up the recovery medical process.

An important step in a computer assisted evaluation (CAE) system for medical images is the segmentation of the lesion, which allows for accurate assessment of the 3D lesion characteristics. Mainly, the purpose of lesion segmentation is to group those pixels that share similar characteristics. In brain MR image, segmentation is the task of dividing brain MR images into regions, aiming to create a resultant segmented image that is more significant and simple to analyse. Lesion segmentation is an exigent task since findings of this sort is typically concealed and embedded in varying densities in intensity of parenchymal tissue structures. A new segmentation method for lesions detection on digital brain MRI images is presented in this paper.
The proposed segmentation method utilizes swarm intelligence approach for segmenting lesion from the brain MR image. Swarm intelligence is an emerging area in the field of optimization and many researchers have developed various algorithms by imitating the behaviours of different swarms of animals and insects such as ants, birds, bees, fishes.

In 1990s, Ant colony optimization algorithm based on ant swarm optimization and Particle swarm optimization algorithms which in turn is based on bird flocks and fish schools from real life has been developed and it has been applied to solve optimization problems in several areas [6]. The Individual agents in swarms displaying a stochastic behavior wherein they behave without any supervision due to her - bee's perception of the neighborhood. Swarms use their environment and resources effectively by collective intelligence [6-7].

The ABC algorithm performs a search for optimum solutions based on concept of the neighborhood search in combination with a random search in a way that models the behavior of a swarm of honey bees in search for their food. The algorithm has been effectively applied to various optimisation problems including wood defect identification [8], the training of neural networks for control chart pattern recognition [9], breast DCE-MRI segmentation [10] and training of neural networks for breast lesion classification [11]. The performance of the swarm bee algorithm is tested on standard classification problems such as diabetes, cancer and heart problems. The classification procedure is done by neural networks and clustering [12]. Recent research indicates that the bee colony algorithm can be used for prolific real world data clustering and mining problems and it is proved that it provides robust performance in comparison to other intelligent optimization methods for a host of complex problems [13-14].

This paper presents a new segmentation algorithm, developed to segment lesions from brain MR images using the Artificial Bee Colony $(\mathrm{ABC})$ clustering algorithm. The remaining of the paper is organized as follows: Section 2 details the basics of MR imaging and the artificial bee colony algorithm. In section 3 exhaustive discussion of the proposed segmentation method for lesion detection and extraction is represented. Section 4 presents the experimental test results and analysis. Section 5 presents the conclusion.

\section{MRI IMAGING}

Magnetic Resonance Imaging (MRI) enables the acquisition of three-dimensional, highly detailed and high resolution images of brain structure, with incomparable soft tissue contrast with respect to other medical imaging methods [15]. The magnetic resonance images are extensively used not only for identifying tissue deformities such as cancers and injuries, but also for studying pathology of brain [16]. In any MR image there exists different types of tissues each with the 
characteristic T1, T2 decay times and proton densities (PD). For instance, the $\mathrm{T} 1$ images give anatomical details, but tend to be noisy due to the short acquisition time $(<1000 \mathrm{~ms}$ for one slice). T2 images possess bigger contrast between the tissues but take longer to acquire $(3000-4000 \mathrm{~ms})$. The PD images (typical acquisition time: $2000 \mathrm{~ms}$ ) generally manifest the smallest contrast between the tissues. Hence PD images present the greatest challenges for anatomical segmentation. The complexity of tissue boundaries causes several voxels to be composed of at least more than two tissues. On the other hand, the constitution of a brain structure cannot be limited to only three tissues (gray matter (GM), white matter (WM), Cerebrospinal fluid (CSF)). If these T1, T2 and PD tissues parameters are calculated from the MR images, the corresponding tissue type can be identified irrespective of its hardware characteristics. Calculated T1, T2 and PD images provides consistent voxel intensity corresponding to the same tissue and therefore it is easier to utilize in segmentation algorithms.

\subsection{Artificial Bee Colony Algorithm}

In the Artificial Bee Colony (ABC) algorithm, the colony of artificial bee consists of three classes of bees: employed, onlooker and scout bees. In ABC algorithm each sequence of food source position (optimal solution ) search consists of three major steps: (1) the employed bees are placed onto the food sources positions (solutions) and then calculating the amounts of nectar (fitness) present in them; (2) selecting the best food source positions (solutions) with the help of onlooker bees after sharing the information acquired from the employed bees and having determined the fitness value of the food sources; (3) identifying the scout bees and placing them at the randomly found out food source positions. In the ABC algorithm, the food position constitutes a possible solution for the optimization problem and the nectar amount of that particular food position is the quality (fitness) of that solution [17].

During initialization phase, generate randomly a set of food source positions (solutions) i.e. a set of employed bee population is randomly produced and the nectar amounts (fitness) values that correspond to them are calculated. Then each employed bee searches around her food source position for identifying a new solution within the neighbourhood of the present solution, and then its associated nectar amount (fitness value) is calculated. If the fitness value of the newly found food source position is higher than the old one, it replaces old one with the new food source position. Else the position of the old one is retained. The employed bees after finishing their search process around the neighbourhood, share the fitness values of the best solutions and they also shared their food source position details with the onlooker bees. The onlooker bees select a higher probability food source position based on their nectar amounts. Each onlooker selects a new food source position within its neighborhood and calculates its nectar amount (fitness). If a particular food source position cannot be improved by a certain number of trails, which is., limit, it means that its associated food source position (solution) has been exhausted by the bees and then the employed bee of this food source position becomes a scout bee and that food source position is said to be unused food source. The scout bee position is replaced with a randomly produced food source position or solution. Until the termination criteria are satisfied these algorithm procedures are repeated [10, 11, 17-19].

\section{IMPLEMENTATION}

The main objective work is to design a new segmentation algorithm for the detection of lesion regions present in brain MRI.

\subsection{Preprocessing}

The pre-processing phase is the most significant part of an image processing system. This phase will apply techniques for removing the noise or improving contrast enhancement of the image. During segmentation task of brain MR image data there exist three main pitfalls which are: image noise, partial volume effects (where more than one tissue is constituted inside a voxel volume) and intensity inhomogeneity [2, 20], which induces spatial changes in tissue static. The use of median filter in the preprocessing phase reduces the effects of random noise, whereas at the same time aids in minimizing the loss of resolution and make the preprocessed image suitable for further processing $[10,21]$.

\subsection{Clustering of Brain MRI Image Using Artificial Bee Colony Algorithm}

Clustering is a method for finding groups that present similarities in the given data, called clusters. The similarity measures are essential element in majority of the clustering algorithms. The most widely used method of evaluating a similarity measure is to make use of distance measures. The Euclidean distance defined in equation (1) is the commonly used distance measure, where $x i(j)$ is the ith data point belonging to the jth cluster, $\mathrm{cj}$ is the jth cluster centre, $\mathrm{m}$ indicates the number of clusters and $n$ is the number of data points in cluster $\mathrm{j}$ [1]. Unsupervised methods do not involve any human interference and can segment the brain MR image with high accuracy. For this reason, unsupervised clustering methods are used over conventional methods.

$$
E=\sum_{j=1}^{m} \sum_{i=1}^{n}\left\|x_{i}^{j}-c_{j}\right\|
$$

Swarm intelligence is a novel distributed intelligence model for solving optimization problems, which has actually taken its inspiration from real life biological examples making use of the swarm concept. The bee colony algorithm based clustering method utilizes the bees searching ability for food source. The main objective is to search for cluster centers (c1, $\mathrm{c} 2, \ldots, \mathrm{cm})$ such that the clustering distance measure defined in Equation (1) is minimised.

\section{The basic steps of $A B C$ clustering operation are:}

Step 0: Start.

Step 1: Initialize the population of solutions $\mathrm{Xi}$ where $\mathrm{i}=1 \ldots . \mathrm{SN}$.

Step 2: Evaluate the population fitness. By employing Equations (2) and (3).

$$
\begin{aligned}
& \text { fit }_{i}=\left\{\begin{array}{ll}
\frac{1}{1+f_{i}} & \text { if } f \geq 0 \\
1+\operatorname{abs}\left(f_{i}\right) & \text { if } f<0
\end{array}\right\} \\
& f=\sum_{i=1}^{S N} 100\left(x_{i}-x_{i}^{2}\right)+\left(x_{i}-1\right)^{2}
\end{aligned}
$$

Step 3: cycle=1 
Step 4: until stopping criteria not met repeat steps 5-9.

Step 5: Generate the new solutions.

Step 6: Select sites for neighborhood search by means of information provided in the

neighborhood of the present one.

Step 7: Recruit bees for the sites that have been selected and calculate the probability by the use of Equation (4).

$$
P_{i}=\frac{f_{i t}}{\sum_{i=1}^{S N} f_{i t}}
$$

Step 8: Select the bee which has better fitness value from each site.

Step 9: The remaining bees in each site are assigned to a random search in the neighborhood and their fitness values are evaluated.

Step 10: cycle $=$ cycle +1

End While (Maximum number of cycles is reached)

Step 11: Stop.

In the proposed $\mathrm{ABC}$ algorithm for clustering brain $\mathrm{MR}$ images, each bee represents a possible clustering solution as set of $\mathrm{M}$ cluster centres, for clustering the brain-MR image four clusters are adequate to discriminate between tissues such as white matter, gray matter, Cerebrospinal fluid, and the lesion or mass, then the $\mathrm{M}$ value can be assigned to four. In $\mathrm{ABC}$ algorithm each site represents a data object. The parameters needed to formulate the algorithm are: employed bee population, number of best sites among selected sites, number of sites chosen for neighbourhood search, population of bees recruited for the best sites, population of bees recruited for the other selected sites and the termination criterion for the loop.

In the initialization phase, population of employed bee is generated randomly to define the $\mathrm{M}$ clusters. The Euclidean distances between each input data of image and all cluster centers are calculated in order to determine to which cluster the input data of image belongs. The preliminary cluster centers are constructed. Having created the cluster centers, the original cluster centers are replaced with the actual centers of the clusters to obtain a particular clustering solution. This initialization procedure is applied, whenever new population of bees is created.

In step 2, the fitness evaluation process is done for each site visited by a bee by using Equations ( 2 and 3); the Rosenbrock function is used to evaluate the fitness. Step 5, is the key step of bee colony optimization algorithm, the new population of bees are produced. In step 6 , the solutions which possess the highest fitness values are selected for a neighborhood search. In step 7 , the bees conduct a search around the selected solutions. The fitness values associated with the solutions are evaluated to determine which solutions are the best. The probability of selected solution is calculated using Equation (4). In step 8, the highest fitness value bees are selected for generating the new bee population. In step 9, new potential solutions (food source positions) are found out by randomly allocating the remaining bees in the population throughout the search space that is available. At the end of each iteration, the population of the bee colony will be split into two groups: the selected bees are included in the next bee population and the scout bees, which are allocated to do random searches for food source position with highest fitness value. These steps are repeated until the termination criterion is met. The performance of this proposed algorithm is evaluated using the parameters extracted from the algorithm output, which are the mean of the fitness function value and the standard deviation of the objective function $[10,11]$.

The artificial bee colony algorithm clustered image is then edge enhanced by unsharp filter which strengthen the image edges through a technique which subtracts an unsharp, or smoothed, version of an image from the given input image.

The segmentation used for extraction of masses or region of interest in brain MR images is the region-based segmentation. In this approach, segmentation of the region is achieved by identifying all pixels that belong to the region based on the intensity of pixels. This work employs region segmentation based on thresholding $[22,23]$. The threshold value selection is done by investigating with numerous threshold values and the pre-eminent threshold value has been selected for extraction of the lesion or region of interest (ROI) from the edge enhanced image. As a result of segmentation, the region matching lesion is extracted from the corresponding input brain MR image.

\section{EXPERIMENTAL RESULTS AND ANALYSIS}

The visual and statistical results of applying the artificial bee colony clustering algorithm for segmenting the mass or lesion and to locate the region of interest (ROI) of the given brain MR image dataset are presented in this section.

\subsection{Evaluation data and methods}

The aim of the experiment is to evaluate the performance of the proposed artificial bee colony clustering based segmentation algorithm over brain MR images received from the Radiology Department of Kovai Medical Center and Hospital (KMCH), Coimbatore, Tamil Nadu, India. The dataset examined is brain MR images acquired under different spatial-temporal resolutions from a 1.5 Tesla MR scanner and the brain MR images obtained from the online source (http://cancerimagingarchive.net.), were also used. Examination of these datasets helps to demonstrate that the present work based segmentation approach to brain MRI analysis is reliable, robust and tolerant of different imaging protocols.

The proposed segmentation method utilizes the ABC algorithm to search for optimum vales for the set of $\mathrm{M}$ cluster centres that minimizes a given clustering metric. The bee colony algorithm converges to the maximum or minimum optimum value without being trapped in local optima. Table 1 presents the parameter values used in the artificial bee colony clustering algorithm implementation, which are decided empirically. The abnormal (lesion) brain MR image datasets were used for testing the proposed algorithm to evaluate its performance. 


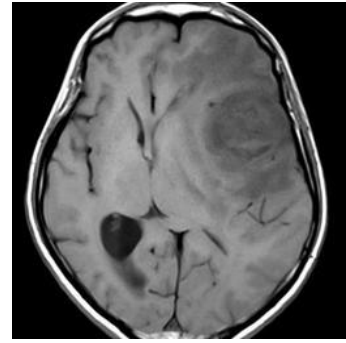

Input image

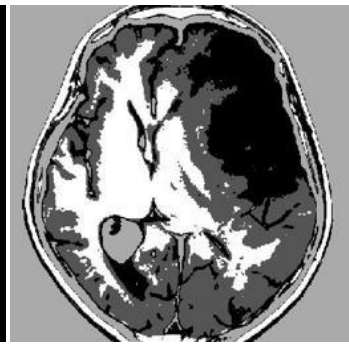

Clustering output

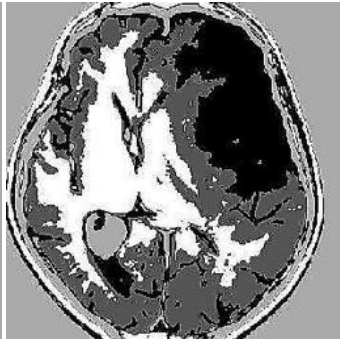

Edge enhanced output

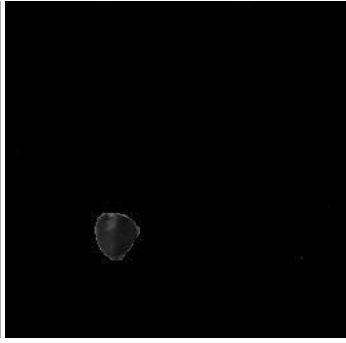

Segmented Lesion

Figure 1 Artificial swarm bee colony clustering based segmentation algorithm output.

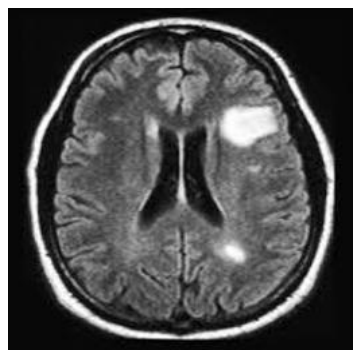

Input image

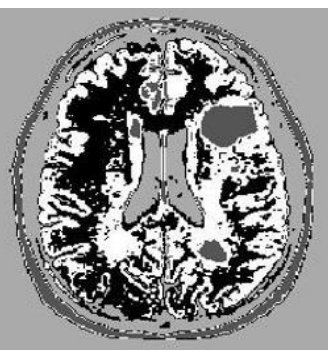

Clustering output

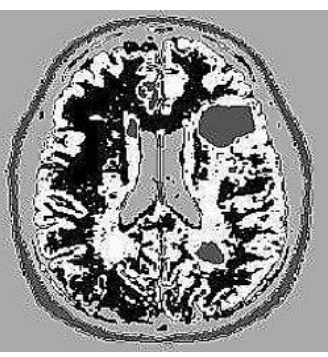

Edge enhanced output

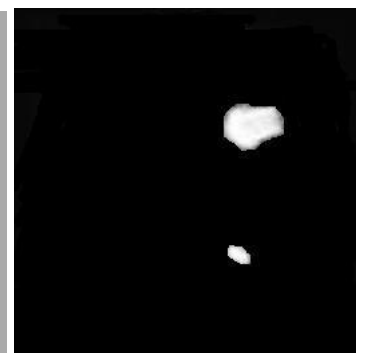

Segmented Lesion

Figure 2 Artificial swarm bee colony clustering based segmentation algorithm output.

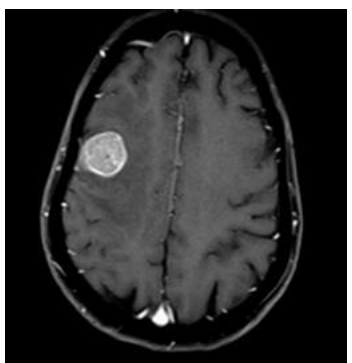

Input image

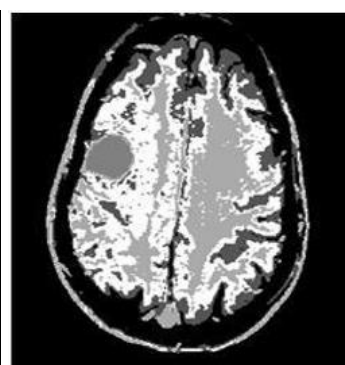

Clustering output

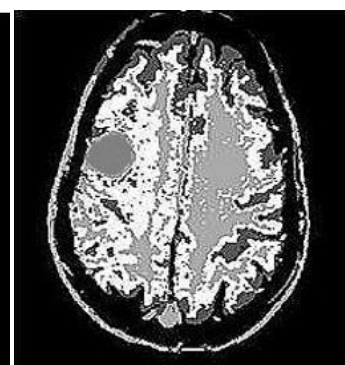

Edge enhanced output

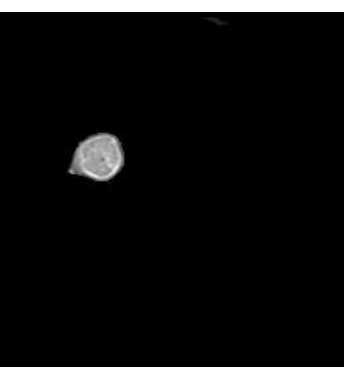

Segmented Lesion

Figure 3 Artificial swarm bee colony clustering based segmentation algorithm output.

The clustering algorithm performance can be assessed in terms of the sensitivity, specificity and accuracy of the system and it is mathematically defined in Equations (5,6 and 7). The brain MR image pairs with lesion were used to evaluate the performance. The sensitivity and accuracy of the proposed segmentation algorithm were evaluated through the comparison of each segmented result with its corresponding ground truth and the ground truth is based on the histopathologist diagnosis. True positive (TP) and false positive (FP) rates are evaluated at different thresholds selected on image pixels in order to calculate the sensitivity and accuracy using Equations (5 \& 7). The sensitivity (true positive detection rate) and 1-specificity (the number of false positive detection rate) are calculated at various threshold values for the segmented images, which are used to evaluate the performance of the algorithm. The threshold value is varied from 0.42 to 0.66 in steps of 0.01 . These rates are calculated and illustrated using ROC curve and the best solution has been plotted as shown in Figure 4,
Sensitivity (SN):

$$
S N=\frac{T P}{T P+F N}
$$

Specificity (SP):

$$
S P=\frac{T N}{T N+F P}
$$

Accuracy,

$$
A C C=\frac{T P+T N}{T P+F P+F N+T N}
$$


The proposed segmentation technique produces consistently higher or equal ROC areas at an input threshold parameter setting of 0.59 and produces reasonable quality ROC areas across the range of parameter values explored. The segmentation results of the proposed algorithm is illustrated in Figures 1 to 3 , which shows a better match between manual division and that of proposed segmentation method achieving a high sensitivity of $98.52 \%$.

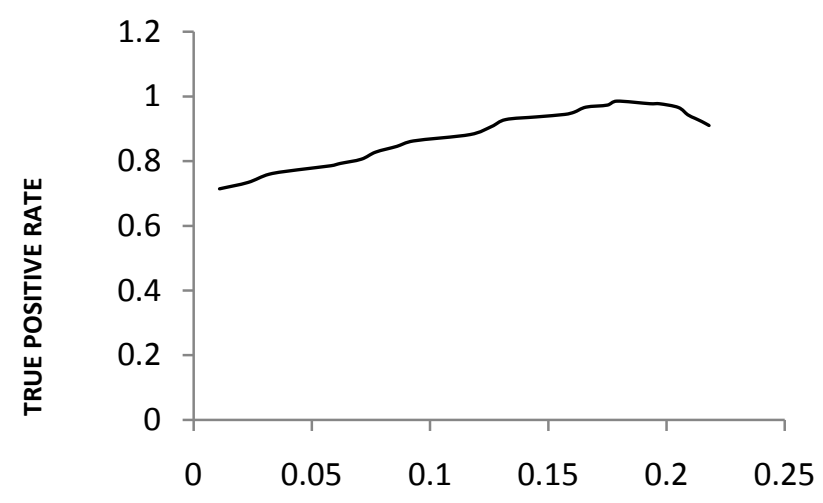

FALSE POSITIVE RATE

Figure 4 The ROC curve of ABC clustering based segmentation.

Table 1 Parameters used in ABC clustering algorithm.

\begin{tabular}{|l|c|}
\hline Parameters & Values \\
\hline Population size of employed bees & 30 \\
\hline Number of sites selected for neighbourhood search & 12 \\
\hline Number of best elite sites out of m selected sites & 4 \\
\hline Population of bees recruited for best e sites & 8 \\
\hline Population of bees recruited for the other selected sites & 3 \\
\hline Limit & 200 \\
\hline Maximum number of iterations, R & 2000 \\
\hline
\end{tabular}

Table 2 Performance measures of ABC clustering based segmentation algorithm.

\begin{tabular}{|c|c|c|c|c|c|}
\hline Image & $\begin{array}{c}\text { Ground } \\
\text { truth } \\
\text { (lesion size } \\
\text { in pixels) }\end{array}$ & $\begin{array}{c}\text { Mean of } \\
\text { fitness } \\
\text { function }\end{array}$ & $\begin{array}{c}\text { Standard } \\
\text { deviation }\end{array}$ & $\begin{array}{c}\text { Lesion } \\
\text { size in } \\
\text { pixels }\end{array}$ & $\begin{array}{c}\text { Accuracy } \\
(\%)\end{array}$ \\
\hline Image 1 & 298 & 0.135117 & 0 & 293 & 98.322 \\
\hline Image 2 & 412 & 0.4150263 & 0 & 397 & 96.359 \\
\hline Image 3 & 336 & 0.0777271 & 0 & 332 & 98.809 \\
\hline
\end{tabular}

\subsection{Performance Evaluation of the} Algorithm

The proposed artificial bee colony clustering segmentation algorithm is evaluated in terms of accuracy, robustness, sensitivity, reliability and efficiency. The performance measures such as standard deviation of objective function (The measure of robustness of a solution), accuracy and mean of fitness function for each segmented image shown in
Figures 1 to 3 are calculated and tabulated in the Table 2. The Rosenbrock function is used to evaluate the fitness of the solution, which is a unimodal function, a function is called unimodal, if it has only one optimum solution. This function leads to faster convergence of the algorithm towards global optimum solution. The mean of fitness function measure indicates searching quality of optimum solution (smaller the mean of fitness function measure more optimum the solution is). The sensitivity of the algorithm is high since it possesses higher accuracy. The swarm bee colony clustering is a robust algorithm and the indication of this fact can be seen from the standard deviations of the results obtained in Table 2.

The robustness of this algorithm can be assured by testing the algorithm over different brain MR image datasets, which results in the use of brain image obtained from different imaging protocol for segmentation as illustrated in Figure 2, where the brain MR image from online source is used to prove that this algorithm is robust to variability of images. This proposed segmentation algorithm is capable to providing the same partition of given input image in all runs which makes it more reliable. The efficiency of the proposed algorithm is tested by measuring the time required for executing the algorithm, it takes 122 seconds and the algorithm occupies very less space. The algorithm is efficient. Further, the proposed bee colony segmentation algorithm can be used for the study of brain pathologies, in particular to brain lesion diagnosis and follow-up. Thus, this algorithm act as a potential tool that can assist the physician with the task of identifying suspicious tissue accurately and faster than can be done by the current manual practice. In addition, the regions detected by the algorithm can be used as input to a computerassisted evaluation system for generating quality features for automatic classification of suspicious tissue.

\section{CONCLUSIONS}

In the proposed new segmentation approach, artificial swarm bee colony algorithm is used for detecting lesion from brain MR images. This approach employs the swarm bee optimization to search for the set of optimum cluster centers. The resultant solution converges to the maximum or minimum optimum value without being trapping at local optima. The Segmented lesion can be further used by the physicians for diagnosing the type of lesion. The evaluation results show that the swarm bee algorithm can be effectively used as an optimization tool for segmenting any type of medical images.

\section{ACKNOWLEDGMENTS}

The author would like to thank Dr R. Rupa, DMRD, DNB, Consultant Radiologist, Kovai Medical Centre and Hospital, Coimbatore, Tamil Nadu, India, for providing the brain MR images along with the ground truth used for testing the algorithm proposed and her valuable clinical advice.

\section{REFERENCES}

[1] Balafar M A, Ramli A R, Saripan M I, Mashohor S. Review of brain MRI image segmentation methods. Artificial Intelligence Review 2010; 33: 261-274.

[2] Janaki Sathya D, Geetha K. A novel clustering based segmentation of multispectral magnetic resonance images. International Journal of Advanced Research in Computer Science 2010; 1: 337-342.

[3] Lee M E, Kim S H, Cho W H. Segmentation of brain MR images using an Ant Colony Optimization algorithm. In: IEEE 2009 Bioinformatics and 
Bioengineering Conference; 22- 24 June 2009; Taichung, Taiwan: IEEE. pp. 366-369.

[4] Ghassabeh YA, Forghani N, Forouzanfar M, Teshnehlab M. MRI fuzzy segmentation of brain tissue using IFCM algorithm with genetic algorithm optimization. In: IEEE 2007 Computer Systems and Applications Conference; 13 -16 May 2007: IEEE. pp. 665-668.

[5] Forghani N, Forouzanfar M, Forouzanfar E. MRI fuzzy segmentation of brain tissue using IFCM algorithm with particle swarm optimization. In: 22nd IEEE 2007 International Symposium on Computer and Information Sciences; 7 - 9 November 2007; Ankara, Turkey: IEEE. pp. 1-4.

[6] Karaboga D, Akay B. A survey: algorithms simulating bee swarm intelligence. Artificial Intelligence Review 2009; 31: 61-85.

[7] Saab S M, El-Omari N K T, Hussein H O. Developing optimization algorithm using artificial bee colony system. Ubiquitous Computing and Communication Journal 2009; 4: 391-396.

[8] Pham DT, Soroka A, Ghanbarzadeh A, Koç E, Otri S, Packianather M S. Optimising neural networks for identification of wood defects using the Bees algorithm. In: IEEE 2006 Industrial Informatics Conference; 16 18 August 2006; Singapore: IEEE. pp. 1346-1351.

[9] Pham DT, Ghanbarzadeh A, Koç E, Otri S. 2006a. Application of the Bees algorithm to the training of radial basis function networks for control chart pattern recognition. In: 5th CIRP 2006 International Seminar on Intelligent Computation in Manufacturing Engineering; 1 January 2006; Ischia, Italy: CIRP. pp. 711-716.

[10] Janaki Sathya D, Geetha K. Development of intelligent system based on artificial swarm bee colony clustering algorithm for efficient mass extraction from breast DCEMR images. International Journal of Recent Trends in Engineering and Technology 2011; 6: 82-88.

[11] Janaki Sathya D, Geetha K. Mass classification in breast DCE-MR images using an artificial neural network trained via a bee colony optimization algorithm. ScienceAsia Journal 2013; 39: 294-305.

[12] Karaboga D, Ozturk C. Fuzzy clustering with artificial bee colony algorithm. Scientific Research and Essays 2010; 5: 1899-1902.

[13] Saeedi S, Samadzadegan F, El-Sheimy N. Object extraction from lidar data using an artificial swarm bee colony clustering algorithm. In: City Models, Roads and Traffic: (CMRT09) ISPSR Workshop: Object Extraction for 3D City Models, Road Databases and Traffic Monitoring - Concepts, Algorithms and Evaluation; 3-4 September 2009; Paris, France: CMRT. pp. 133-138.

[14] Pham DT, Ghanbarzadeh A, Koç E, Rahim S, M. Zaidi M. The bee's algorithm - A novel tool for complex optimization problems. In: 2nd Virtual International Conference on Intelligent Production Machines and Systems (IPROMS-06); 3 - 14 July 2006; Cardiff, UK: IPROMS. pp. 454-459.

[15] Atkins M S, Mackiewich B T. Fully automatic segmentation of the brain in MRI. IEEE Transaction on Medical Imaging 1998; 17: 98-107.

[16] Cocosco C A, Zijdenbos A P, Evans, A C. A fully automatic and robust brain MRI tissue classification method. Medical Image Analysis 2003; 7: 513-527.

[17] Karaboga D, Basturk B. A powerful and efficient algorithm for numerical function optimization: artificial bee colony $(\mathrm{ABC})$ algorithm. Journal of Global Optimization 2007; 39: 459-471.

[18] Karaboga D. An idea based on honey bee swarm for numerical optimization. Technical report-TR06; engineering faculty, computer engineering department, Erciyes University; October 2005; Kayseri, Turkey, pp. $1-10$.

[19] Basturk B, Karaboga D. An artificial bee colony (ABC) algorithm for numerical function optimization. In: IEEE 2006 Swarm Intelligence Symposium; 12-14 May 2006; Indianapolis, Indiana, USA: IEEE. pp. 1-9

[20] Wells W M, Grimson W E L, Kikins R. Adaptive segmentation of MRI data. IEEE Transaction on Medical Imaging 1996; 15: 429-442.

[21] Janaki Sathya D, Geetha K. An optimized preprocessing decision for multispectral MRI- based applications. International Journal of Advanced Research in Computer Science, 2(4), 386-391, 2011.

[22] Weszka J S. A Survey of threshold selection techniques. Computer Graphics and Image Processing 1978; 7: 259 265

[23] Janaki Sathya D, Geetha K. Comparative study of different edge enhancement filters in spatial domain for magnetic resonance images. AMSE Signal Processing and Pattern Recognition Journal 2011; 54: 30-43. 